\title{
Associations of Serum Lipids with Risk of Cognitive Impairment in Chinese Centenarians
}

\section{Yujian Chen ( $\nabla$ triser@126.com )}

Hainan Hospital of PLA General Hospital https://orcid.org/0000-0001-5027-661X

\section{Ya Huang}

Hainan Hospital of PLA General Hospital

\section{ChaoXue Ning}

Hainan Hospital of PLA General Hospital

Qiao Zhu

Hainan Hospital of PLA General Hospital

\section{Yao Yao}

Center for Healthy Aging and Developmemt studies, National School of Development, Peking University

\section{Yali Zhao}

Hainan Hospital of PLA General Hospital

\section{Research article}

Keywords: Cognitive impairment, Serum lipids, Centenarian

Posted Date: March 2nd, 2021

DOI: https://doi.org/10.21203/rs.3.rs-274807/v1

License: (c) (1) This work is licensed under a Creative Commons Attribution 4.0 International License.

Read Full License 


\section{Abstract \\ Background}

Dyslipidaemia and cognitive impairment are common in old adults and risks of both of them increase with increasing age. However, the relationship between lipids and cognitive impairment in longevous people is still unclear. This study aimed to assess the association between serum lipids and cognitive impairment in Chinese centenarians.

\section{Methods}

In a cross-sectional study, total cholesterol (TC), triglycerides (TG), high-density lipoprotein cholesterol (HDL-C), and low-density lipoprotein cholesterol (LDL-C) were biochemically measured in 924 centenarians and cognitive functions were evaluated. Multiple logistic regression analysis was used to examine the associations of lipids with cognitive impairment and the risks for cognitive impairment associated with quartile of serum lipid concentrations.

\section{Results}

The results demonstrated HDL-C was an independent beneficial factor for cognitive impairment after controlling for the potential covariates (Odds ratio, $\mathrm{OR}=0.350,95 \%$ Confidence interval, $\mathrm{Cl}=0.159-0.774$; $P=0.009)$. An inverse association of HDL-C with cognitive impairment was found ( $P$ for trend $=0.001)$ and the association remained significant after adjusting for possible cofounders ( $P$ for trend $=0.012$ ). The OR of cognitive impairment for the lowest versus highest quartiles of HDL-C was $1.96(95 \% \mathrm{Cl}, 1.09-$ 3.45).

\section{Conclusions}

The results indicated that low HDL-C is likely to be harmful for cognitive functions in centenarians. The cognitive function of longevous persons with low HDL-C concentrations deserves more attention.

\section{Background}

Cognitive impairment, ranging from mild deficits to dementia, is a common neurodegenerative disease in older adults [1,2]. The incidence of cognitive impairment increases exponentially with increasing age. The incidence of dementia is $40.5 \%$ among the elderly aged 90 and above in China, whereas it is $1.5 \%$ in those aged 60-64 [3].

Lipids plays a significant role in neuronal development, synaptic plasticity and brain function [4-6]. Cholesterol alters the degradation of the amyloid precursor protein in brain tissue, which plays a major 
role in the pathogenesis of Alzheimer's disease (AD) [7, 8]. The disruption of lipid homeostasis could therefore be an important factor in the pathogenesis of cognitive impairment $[9,10]$.

Several studies have investigated the relationship between serum lipids and cognitive impairment among the elderly adults, but have yielded conflicting results. Exel, E.V. et al. reported that HDL-C was negatively associated with cognitive impairment in elderly general population $[10,11]$. Ma, C. et al. found that TC and LDL-C were risk factors for cognitive functions and HDL-C was not $[12,13]$. Anstey, K. J. et al. claimed that late-life measured TC and HDL-C were not associated with risk of mild cognitive impairment, $A D$, or any dementia [14]. Huang, C.Q. et al. reported that no significant association between serum lipid concentrations with cognitive function in old adults $[15,16]$. Besides, evidences relating to the association of serum lipids with cognitive function in centenarians are scarce. This study aimed to examine the association between lipid profiles and cognitive impairment in Chinese centenarians. Better knowledge of the relationships between lipids and cognitive function might help decrease the overall incidence of dementia in longevous people.

\section{Methods}

\section{Subjects}

Subjects in this study were obtained from the China Hainan Centenarian Cohort Study (CHCCS)[17], which was a community-based study of inhabitants of Hainan Province, from June 2014 to December 2016. Details of this study including sampling strategy and interview procedures have been described elsewhere [17, 18]. In brief, 1002 inhabitants (822 women and 180 men)) in Hainan aged 100 years or older were interviewed and blood samples were collected. Home interview, physical examination and blood analysis were performed following standard procedures. In this study, the subjects (29 women and 10 men) who were unable to complete interview because of weakness, hearing disorder, speech impediment, poor eyesight or other personal reasons were excluded from the data analyses. 39 participants were excluded because of missing information on serum lipid profiles. 924 centenarians (759 women and 165 men) were included in the final analysis.

All subjects provided written informed consent.

For cognitively impaired subjects, informed consent was obtained from a guardian.

The study protocol was approved by the Ethics Committee of the Hainan Hospital of the Chinese People's Liberation Army General Hospital (Sanya, Hainan).

\section{Cognitive Function}

Cognitive functions of centenarians were evaluated using two methods. One method was using MiniMental State Examination (MMSE) [19], which is a 30-point screening test to quantitatively assess the severity of cognitive impairments. In another method cognitive functions of centenarians were evaluated by general practitioners. On account of MMSE values were too aberrant (Mean MMSE $=9.61,96.2 \%$ MMSE $\leq 23$ ) to group by the general standard (Severe cognitive $<18$, no cognitive impairment $>23,18 \leq$ 
mild cognitive impairment $\leq 23$ ), centenarians was divided into two groups (normal and cognitive impairment) according to the assessment of general practitioners.

\section{Serum lipid analysis}

Venous blood samples collected from each participant were transported in a cold storage $\left(4^{\circ} \mathrm{C}\right)$ to the Clinical Laboratory and were assayed within $6 \mathrm{~h}$. Serum concentration TC, TG, HDL-C and LDL-C were analyzed on a fully automated biochemical analyzer (Roche Cobas c702, Switzerland) using a standard procedure.

\section{Possible Confounders}

Confounder information was obtained from a structured questionnaire for the baseline survey. Some potential confounding factors, for example living habits, may be associated with both cholesterol levels and risk of cognitive impairment. Therefore, several potential confounders were controlled when the relationship between serum cholesterol level and cognitive impairment were assessed. The confounder included demographic characteristics, lifestyle factors, certain foods consumption, physical and blood measurement (Table 1). Participants were dichotomized into illiterate versus primary school or above. Ethnicities were dichotomized into Han (the predominant ethnicity in China) versus non-Han. Smoking, alcohol use, and tea drinking were categorized as current versus former or never. Activity of daily living (ADL) impairment was scored according to Barthel Index [20], and ADL impairment was defined as a score of less than 90 . Outdoor activities were defined as daily outdoor walking or gardening for more than $1 \mathrm{~h}$. Living conditions referred to live with families or not. Social interactions included relatives and friends contacts, which were defined as meet and communicate with at least one relative or friend once a month. Regular consumption was defined as consumption of the food at least once per week.

\section{Statistical Analysis}

Continuous variables with normal distribution were described as the mean and standard deviation. Categorical variables were described as the number and percentage. Continuous variables were compared with Student's t-test (normal distribution) and Mann-Whitney $U$ test (skewed distribution). Categorical variables were compared with Chi-square test. The association between cognitive impairment and serum lipid levels or some covariates was assessed using multiple logistic regression analysis. ORs and $95 \% \mathrm{Cl}$ of cognitive impairment were further evaluated according to quartile of serum lipid concentrations using logistic regression analysis. Adjustments were made for demographic factors, lifestyle factors and regular consumption of certain food listed in Table 1. Two-sided p-values less than 0.05 were considered statistically significant. All statistical analyses were performed with the Statistic Package for Social Science (SPSS) version 22.0 (SPSS Inc., Chicago, USA).

\section{Results}

A total of 924 centenarians ( 759 women and 165 men) were included in this study with self-reported age ranging between 100 and 115 . As Table 1 shows, the majority participants were female (82.1\%), Han 
ethnic (88.1\%) and illiterate (91.8\%). The overall mean (standard deviation, s.d.) plasma concentrations of TC, TG, LDL-C, HDL-C were 4.67 (1.01) mM, 1.18 (0.66) mM, $2.80(0.80) \mathrm{mM}$ and 1.43 (0.39) mM respectively. The mean \pm s.d. MMSE scores was $9.61 \pm 6.29$. The overall prevalence rate of the cognitive impairment among the longevous persons was $38.7 \%(95 \% \mathrm{Cl}, 35.6-41.9 \%)$. 
Table 1

Baseline characteristics of the participants according to cognitive status

\begin{tabular}{|c|c|c|c|c|}
\hline & $\begin{array}{l}\text { Total } \\
\text { cases }\end{array}$ & $\begin{array}{l}\text { No cognitive } \\
\text { impairment }\end{array}$ & $\begin{array}{l}\text { Cognitive } \\
\text { impairment }\end{array}$ & $P$ \\
\hline Number of participants & 924 & 566 & 358 & \\
\hline \multicolumn{5}{|l|}{ Demographic factors } \\
\hline $\begin{array}{l}\text { Age at baseline, mean (s.d.) } \\
\text { (years) }\end{array}$ & $\begin{array}{l}102.8 \\
(2.7)\end{array}$ & $102.8(2.8)$ & $102.7(2.6)$ & 0.627 \\
\hline Female (\%) & 82.1 & 80.4 & 84.9 & 0.080 \\
\hline Illiterate, \% & 91.8 & 90.5 & 93.9 & 0.067 \\
\hline Han ethnic, \% & 88.1 & 88.3 & 87.7 & 0.773 \\
\hline \multicolumn{5}{|l|}{ Lifestyle factors } \\
\hline Current smoker, \% & 3.6 & 3.9 & 3.2 & 0.580 \\
\hline Current alcohol drinker, \% & 11.5 & 12.6 & 9.9 & 0.221 \\
\hline Current tea drinker, $\%$ & 9.9 & 10.7 & 8.7 & 0.318 \\
\hline ADL impairments, \% & 71.3 & 62.7 & 84.9 & $\dot{0} 001$ \\
\hline Outdoor activities > 1 h/d, \% & 58.4 & 67.9 & 43.5 & $\begin{array}{l}< \\
0.001\end{array}$ \\
\hline Living with families, \% & 86.0 & 85 & 87.7 & 0.244 \\
\hline Relatives contacts, \% & 89.9 & 92.3 & 86.2 & 0.003 \\
\hline \multicolumn{5}{|c|}{ Regular consumption of certain foods (\%) } \\
\hline Meat & 86.8 & 87.6 & 85.6 & 0.399 \\
\hline Poultry & 46.8 & 49.5 & 42.6 & 0.044 \\
\hline Fish & 64.6 & 70.6 & 54.9 & $\dot{0.001}$ \\
\hline Milk & 31.3 & 33.8 & 27.4 & 0.043 \\
\hline Eggs & 42.3 & 47.9 & 33.5 & $\begin{array}{l}<.001 \\
0.001\end{array}$ \\
\hline Fresh fruit & 61.9 & 66.7 & 54.5 & $\begin{array}{l}< \\
0.001\end{array}$ \\
\hline Vegetable & 95.4 & 97.3 & 92.4 & $\begin{array}{l}< \\
0.001\end{array}$ \\
\hline
\end{tabular}

SBP, systolic blood pressure; DBP, diastolic blood pressure; BMI, Body mass index. 


\begin{tabular}{|c|c|c|c|c|}
\hline & $\begin{array}{l}\text { Total } \\
\text { cases }\end{array}$ & $\begin{array}{l}\text { No cognitive } \\
\text { impairment }\end{array}$ & $\begin{array}{l}\text { Cognitive } \\
\text { impairment }\end{array}$ & $P$ \\
\hline \multicolumn{5}{|c|}{ Physical and blood measurements, mean (s.d.) } \\
\hline $\mathrm{SBP}(\mathrm{mmHg})$ & $\begin{array}{l}151.0 \\
(23.6)\end{array}$ & $152.6(23.2)$ & $148.7(24.1)$ & 0.030 \\
\hline $\mathrm{DBP}(\mathrm{mmHg})$ & $\begin{array}{l}74.9 \\
(12.2)\end{array}$ & $75.4(11.8)$ & $74.3(12.8)$ & 0.171 \\
\hline $\mathrm{BMI}\left(\mathrm{kg} / \mathrm{m}^{2}\right)$ & $18.2(3.4)$ & $18.5(3.3)$ & $17.6(3.8)$ & 0.001 \\
\hline \multicolumn{5}{|c|}{ Lipid measurements, mean (s.d.) } \\
\hline $\mathrm{TC}(\mathrm{mM})$ & $\begin{array}{l}4.67 \\
(1.01)\end{array}$ & $4.73(0.98)$ & $4.57(1.04)$ & 0.021 \\
\hline $\mathrm{TG}(\mathrm{mM})$ & $\begin{array}{l}1.18 \\
(0.66)\end{array}$ & $1.17(0.57)$ & $1.18(0.80)$ & 0.509 \\
\hline LDL-C (mM) & $\begin{array}{l}2.80 \\
(0.80)\end{array}$ & $2.84(0.80)$ & $2.73(0.80)$ & 0.027 \\
\hline $\mathrm{HDL}-\mathrm{C}(\mathrm{mM})$ & $\begin{array}{l}1.43 \\
(0.39)\end{array}$ & $1.47(0.38)$ & $1.38(0.39)$ & $\begin{array}{l}<.001 \\
0.00\end{array}$ \\
\hline Apolipoprotein B (g/L) & $\begin{array}{l}0.94 \\
(0.26)\end{array}$ & $0.94(0.26)$ & $0.93(0.26)$ & 0.413 \\
\hline Apolipoprotein A (g/L) & $\begin{array}{l}1.38 \\
(0.30)\end{array}$ & $1.42(0.29)$ & $1.33(0.31)$ & 0.000 \\
\hline \multicolumn{5}{|c|}{ Cognitive functions, median (interquartile range) } \\
\hline MMSE score & $8.0(8.0)$ & $10.0(9.0)$ & $5.0(7.0)$ & $\begin{array}{l}<.001 \\
0.001\end{array}$ \\
\hline
\end{tabular}

Table 1 compares the characteristics of participants with and without cognitive impairment. No statistically significant differences were found for demographic factors. Compared with normal cases, cognitive impairment cases had a higher prevalence of ADL impairments $(P<0.001)$, and were less likely to participate in regular outdoor activities $(P<0.001)$ and maintained contact with their relatives $(P=$ 0.003). Participants with cognitive impairment had a very different dietary pattern compared with normal. Regular consumption of certain foods, such as poultry, fish, milk, eggs, fresh fruit and vegetable were less common in cognitive impairment cases than in normal $(P<0.05)$. SBP, BMI, TC, LDL-C, HDL-C, and apolipoprotein A were lower in participants with cognitive impairment than those without $(P S<0.05)$. The distribution of other lifestyle factors and physical measurements were similar.

Table 2 shows the results of the multiple logistic regression analysis. Participants who were with low HDL-C, had ADL impairments or no relative contacts, lacked outdoor activities or frequent fish and eggs 
intake tended to have higher odds of cognitive impairment $\left(P_{S}<0.05\right)$. The OR between HDL-C and cognitive impairment after adjusting for other covariates was $0.350(0.159-0.774, P=0.009)$. No significant associations were found between cognitive impairment and TC, TG or LDL-C.

To further investigate the association between serum lipid concentrations and cognitive impairment in centenarians, the participants were categorized into four groups according to the quartiles of lipid concentrations. As Table 3 shows, a negative association between HDL-C and cognitive impairment was observed (Model 1, $P$ for trend $=0.001$ ). After adjusting for confounding variables, this negative relationship remained statistically significant (Model $2, p$ for trend $=0.012$ ). The multiple adjusted OR of cognitive impairment for the highest versus lowest quartiles of HDL levels was 0.51 (95\% $\mathrm{Cl}, 0.29-0.92)$. No associations were found between cognitive impairment and TG, TC or LDL-C.

\section{Discussion}

In this study, we explored the association between serum lipid profiles and cognitive impairment in China Hainan centenarians. We found that high HDL-C were associated with an decreased prevalence of cognitive impairment, and this association remained significant after adjusting for demographic and other potential confounders. Serum concentrations of TC, TG, and LDL-C showed no association with cognitive impairment. Our results expanded the knowledge that high levels of HDL-C are associated with better cognitive function in centenarian.

Table 2 Significant associates for cognitive impairments by multiple logistic regression 


\begin{tabular}{|lllll|}
\hline & OR & $95 \% \mathrm{Cl}$ & & $P$ \\
\hline TC & 1.612 & 0.798 & 3.255 & 0.183 \\
\hline TG & 0.941 & 0.685 & 1.294 & 0.709 \\
\hline HDL-C & 0.350 & 0.159 & 0.774 & 0.009 \\
\hline LDL-C & 0.598 & 0.273 & 1.308 & 0.198 \\
\hline Age & 0.986 & 0.929 & 1.045 & 0.631 \\
\hline Female & 0.887 & 0.551 & 1.428 & 0.621 \\
\hline Illiterate & 0.742 & 0.395 & 1.393 & 0.354 \\
\hline Han ethnic & 1.429 & 0.819 & 2.493 & 0.209 \\
\hline Current smoker & 0.803 & 0.325 & 1.987 & 0.635 \\
\hline Current alcohol drinker & 1.025 & 0.588 & 1.788 & 0.929 \\
\hline Current tea drinker & 0.807 & 0.286 & 2.280 & 0.686 \\
\hline ADL impairments & 2.283 & 1.533 & 3.400 & 0.000 \\
\hline Outdoor activities $>1$ h/d & 0.485 & 0.346 & 0.681 & 0.000 \\
\hline Living with families & 0.940 & 0.584 & 1.511 & 0.797 \\
\hline Relatives contacts & 0.543 & 0.305 & 0.966 & 0.038 \\
\hline Frequent meat intake & 1.183 & 0.733 & 1.907 & 0.491 \\
\hline Frequent poultry intake & 1.101 & 0.760 & 1.596 & 0.611 \\
\hline Frequent fish intake & 0.553 & 0.393 & 0.779 & 0.001 \\
\hline Frequent milk drinking & 0.963 & 0.642 & 1.443 & 0.854 \\
\hline Frequent eggs intake & 0.617 & 0.421 & 0.905 & 0.013 \\
\hline Frequent fruit intake & 0.832 & 0.579 & 1.196 & 0.321 \\
\hline Frequent vegetable intake & 0.474 & 0.212 & 1.058 & 0.068 \\
\hline SBP & 0.991 & 0.983 & 1.000 & 0.049 \\
\hline DBP & 1.007 & 0.990 & 1.024 & 0.417 \\
\hline BMl & 0.966 & 0.913 & 1.022 & 0.228 \\
\hline
\end{tabular}

Elevated serum HDL-C levels have been shown to be protective against adverse health outcomes in elderly subjects, including cardiovascular disease, stroke and cognitive impairment [10, 11, 21, 22]. Among people aged 80 years or older, higher 
Table 3 ORs for Cognitive Impairment over Strata of Lipid Concentrations

\begin{tabular}{|c|c|c|c|c|c|}
\hline & Q1 & Q2 & Q3 & Q4 & $\begin{array}{l}P \text { for } \\
\text { trend }\end{array}$ \\
\hline \multicolumn{6}{|l|}{ TC } \\
\hline $\begin{array}{l}\text { Mean } \\
\text { (range) }\end{array}$ & $3.47(1.34-4.02)$ & $\begin{array}{l}4.33(4.03- \\
4.58)\end{array}$ & $4.90(4.59-5.26)$ & $\begin{array}{l}6.01(5.27- \\
8.85)\end{array}$ & \\
\hline Model 1 & 1.00 (Reference) & $\begin{array}{l}1.03(0.64 \\
1.67)\end{array}$ & $\begin{array}{l}1.63(0.88 \\
3.02)\end{array}$ & $\begin{array}{l}1.20(0.53 \\
2.72)\end{array}$ & 0.285 \\
\hline Model 2 & 1.000(Reference) & $\begin{array}{l}0.86(0.48 \\
1.56)\end{array}$ & $\begin{array}{l}1.49(0.71 \\
3.15)\end{array}$ & $\begin{array}{l}1.22(0.46, \\
3.22)\end{array}$ & 0.348 \\
\hline \multicolumn{6}{|l|}{ TG } \\
\hline $\begin{array}{l}\text { Mean } \\
\text { (range) }\end{array}$ & $0.66(0.34-0.80)$ & $\begin{array}{l}0.91(0.81- \\
1.03)\end{array}$ & $1.20(1.04-1.40)$ & $\begin{array}{l}1.18(1.41- \\
9.99)\end{array}$ & \\
\hline Model 1 & 1.00 (Reference) & $\begin{array}{l}0.73(0.49 \\
1.07)\end{array}$ & $\begin{array}{l}0.82(0.55 \\
1.21)\end{array}$ & $\begin{array}{l}0.74(0.48 \\
1.14)\end{array}$ & 0.332 \\
\hline Model 2 & 1.00 (Reference) & $\begin{array}{l}0.64(0.40 \\
1.02)\end{array}$ & $\begin{array}{l}0.90(0.56 \\
1.45)\end{array}$ & $\begin{array}{l}0.87(0.52, \\
1.47)\end{array}$ & 0.959 \\
\hline \multicolumn{6}{|l|}{ HDL } \\
\hline $\begin{array}{l}\text { Mean } \\
\text { (range) }\end{array}$ & $0.98(0.50-1.17)$ & $\begin{array}{l}1.28(1.18- \\
1.39)\end{array}$ & $1.52(1.40-1.67)$ & $\begin{array}{l}1.97(1.68- \\
2.87)\end{array}$ & \\
\hline Model 1, & 1.00 (Reference) & $\begin{array}{l}0.67(0.46 \\
0.98)\end{array}$ & $\begin{array}{l}0.50(0.33, \\
0.76)\end{array}$ & $\begin{array}{l}0.49(0.30 \\
0.78)\end{array}$ & 0.001 \\
\hline Model 2 & 1.00 (Reference) & $\begin{array}{l}0.69(0.44 \\
1.10)\end{array}$ & $\begin{array}{l}0.553(0.34, \\
0.91)\end{array}$ & $\begin{array}{l}0.51(0.29 \\
0.92)\end{array}$ & 0.012 \\
\hline \multicolumn{6}{|l|}{ LDL } \\
\hline $\begin{array}{l}\text { Mean } \\
\text { (range) }\end{array}$ & $1.88(0.64-2.28)$ & $\begin{array}{l}2.48(2.29- \\
2.72)\end{array}$ & $2.97(2.73-3.25)$ & $\begin{array}{l}3.87(3.26- \\
6.76)\end{array}$ & \\
\hline Model 1 & 1.00 (Reference) & $\begin{array}{l}0.99(0.63 \\
1.56)\end{array}$ & $\begin{array}{l}0.51(0.28 \\
0.91)\end{array}$ & $\begin{array}{l}0.66(0.31 \\
1.40)\end{array}$ & 0.057 \\
\hline Model 2 & 1.00 (Reference) & $\begin{array}{l}1.25(0.72 \\
2.17)\end{array}$ & $\begin{array}{l}0.72(0.36 \\
1.46)\end{array}$ & $\begin{array}{l}0.80(0.32, \\
1.97)\end{array}$ & 0.274 \\
\hline
\end{tabular}

Mean (range): mmol/L; Model 1 and Model 2: OR (95\% Cl).

Model 1: No adjusted. Model 2: Adjusted for the potential covariates listed in Table 2.

HDL-C concentration was independently associated with better functional status, physical performance and survival $[23,24]$. Serum HDL-C was found to be a protective predictor of executive function in older 
patients with diabetes mellitus[25]. Lower HDL-C concentration was associated with cognitive impairment and clinical diagnosis of dementia in the oldest old [10]. In our study, high HDL-C levels were found to be associated with lower adjusted odds $(\mathrm{OR}=0.51)$ of cognitive impairment. This result is in accordance with previous studies.

Several studies reported that TC and LDL-C are related to cognitive impairment in China old adults [12, 13]. In our study, no associations were found between TC, TG and LDL-C and cognitive impairment in China centenarians. Further studies are required to explore the possible roles of TC and LDL-C in maintaining cognitive function in the oldest-old.

There are some hypotheses that may explain the observed association between HDL-C and cognitive impairment. First, HDL-C prevents atherosclerosis progression and cause regression of established atherosclerotic lesions through its reverse cholesterol transport capacity [26]. Increasing HDL-C concentration reduces the risk for atherosclerotic disease and stroke which are important risk factors for cognitive impairment [27]. Second, HDL-C has effects of antioxidant, anti-inflammatory, inhibition of platelet aggregation, and improvement of endothelial function [26]. Those effects are also critical in atheroprotective progression. Third, HDL cholesterol is the predominant lipoprotein in the human brain, where it can prevent aggregation of amyloid- $\beta$ peptide and the development of neurodegeneration $[7,28]$. This might slow or even prevent the development of cognitive impairment.

Age is a significant risk determinant of cognitive impairment $[3,29]$ and the incidence of severe cognitive impairment continues to rise exponentially after the age of 90 [30]. In this study, the incidence of cognitive impairment in China centenarians was $38.7 \%(95 \% \mathrm{Cl}, 35.6-41.9 \%)$. Given that $\mathrm{HDL}-\mathrm{C}$ is a protective factor for cognitive impairment, normalization of HDL-C levels may become a future strategy to prevent the development of cognitive impairment in long-lived persons. The change of blood HDL-C concentration may be as an effective indicator for cognitive functions change in elderly persons. These hypotheses should be further tested in appropriately designed, randomized, controlled trials.

While highlighting the strengths of our study, several limitations must be mentioned. First, this study was cross-sectional designed, and thus causality could not be inferred. Further longitudinal studies are needed to confirm the association between HDL-C and cognitive impairment in the Chinese oldest-old. Second, the diagnosis of cognitive impairment were not confirmed by clinical neurologists and errors were exist possibly in the detection by general practitioners. Therefore, the effects of HDL-C, TC, TG and LDL-C on cognitive impairment might be overestimated or underestimated in this study. Although MMSE is a widely used test in evaluation of cognitive function, it is problematic when specifically used in examining cognitive performance in oldest old participants [31,32]. MMSE scores of centenarians were easily influenced by health problems, illiteracy (the majority of Hainan centenarians received no education), sensory impairment and physical disability, and might not reflect the actual level of cognitive function in centenarians. Objective and practical methods should be developed to evaluate the cognitive functions of centenarians. Third, our subjects are unlikely to be completely representative of the entire China 
centenarians, because they were all recruited from Hainan Province which is an independent sea island area and has a low proportion of immigrants.

\section{Conclusions}

In conclusion, serum HDL-C concentrations were independently and negatively associated with cognitive impairment in Chinese oldest-old. Centenarians with high HDL-C were likely to have better cognitive function. Further longitudinal studies with larger sample size, and a comprehensive neuropsychological diagnosis to capture specific cognitive functions are warranted to confirm our findings.

\section{Abbreviations}

TC: Total Cholesterol; TG: Triglycerides; HDL-C: High-Density Lipoprotein Cholesterol; LDL-C: Low-Density Lipoprotein Cholesterol; OR: Odds Ratio; Cl: Confidence interval; AD: Alzheimer's Disease; CHCCS: the China Hainan Centenarian Cohort Study; MMSE: Mini-Mental State Examination; ADL: Activity of Daily Living ; SPSS: Statistic Package for Social Science $₫$ s.d.: standard deviation $\llbracket$ SBP: Systolic Blood Pressure; DBP: Diastolic Blood Pressure; BMI: Body Mass Index.

\section{Declarations}

\section{Ethics approval and consent to participate}

Written informed consent was obtained from all men and women who participated in the CHCCS study. The Ethics Committee of the Hainan Hospital of the Chinese People's Liberation Army General Hospital (Sanya, Hainan; No. 301hn11201601) approved the study protocol.

\section{Consent for publication}

Written informed consent for publication was obtained from all participants.

\section{Availability of data and materials}

The data supporting this study is available from the corresponding author for reasonable request.

\section{Competing interests}

The authors declare that they have no competing interests.

\section{Funding}

This work was supported by grants from National Natural Science Foundation of China (grant nos. 81801251, 81941021), National Key Research and Development Program of China (grant nos. 2018YFC2000400), National Scientific and Technological Resource Sharing Service Platform Project of China (grant nos. YCZYPT201807), Medical Big Data Research and Development Program of PLA 
General Hospital (grant nos. MBD2018030). There is no role of the funding body in the design of the study and collection, analysis, and interpretation of data and in writing the manuscript.

\section{Authors' contributions}

YJC and YH conceived and designed the project, draft and revised the paper; YJC, YH, CXN, QZ, and YY collected the data and conducted the data analysis; YJC, YH and YLZ supervised the analyses and suggested revisions of the paper. All the authors have read and approved the final manuscript.

\section{Acknowledgements}

Not applicable.

\section{References}

1. Montine, T.J., S.A. Bukhari, and L.R. White, Cognitive Impairment in Older Adults and Therapeutic Strategies. Pharmacol Rev, 2021. 73(1): p. 152-162.

2. Cai, Z. and H. Li, An Updated Review: Androgens and Cognitive Impairment in Older Men. Frontiers in Endocrinology, 2020. 11.

3. World Alzheimer Report 2015. The global impact of dementia: An analysis of prevalence, incidence, cost and trends. Available at: https://www.alz.co.uk/research/WorldAlzheimerReport2015.pdf. 2015.

4. Hayashi, H., Lipid metabolism and glial lipoproteins in the central nervous system. Biol Pharm Bull, 2011. 34(4): p. 453-61.

5. Mulder, M., Sterols in the central nervous system. Curr Opin Clin Nutr Metab Care, 2009. 12(2): p. 1528.

6. Pfrieger, F.W., Cholesterol homeostasis and function in neurons of the central nervous system. Cell Mol Life Sci, 2003. 60(6): p. 1158-71.

7. Habchi, J., et al., Cholesterol catalyses Abeta42 aggregation through a heterogeneous nucleation pathway in the presence of lipid membranes. Nat Chem, 2018. 10(6): p. 673-683.

8. Wood, W.G., et al., Cholesterol as a causative factor in Alzheimer's disease: a debatable hypothesis. J Neurochem, 2014. 129(4): p. 559-72.

9. Banerjee, S. and S. Mukherjee, Cholesterol: A Key in the Pathogenesis of Alzheimer's Disease. ChemMedChem, 2018. 13(17): p. 1742-1743.

10. Exel, E.v., et al., Association between high-density lipoprotein and cognitive impairment in the oldest old. Ann Neurol, 2002. 51(6): p. 716-21.

11. Choi, H., et al., Serum high-density lipoprotein cholesterol concentration and functional state: The Korean Urban Rural Elderly (KURE) Study. Arch Gerontol Geriatr, 2017. 71: p. 115-121.

12. Ma, C., et al., Blood cholesterol in late-life and cognitive decline: a longitudinal study of the Chinese elderly. Mol Neurodegener, 2017. 12(1): p. 24. 
13. Zhou, F., et al., High Low-Density Lipoprotein Cholesterol Inversely Relates to Dementia in CommunityDwelling Older Adults: The Shanghai Aging Study. Front Neurol, 2018. 9: p. 952.

14. Anstey, K.J., K. Ashby-Mitchell, and R. Peters, Updating the Evidence on the Association between Serum Cholesterol and Risk of Late-Life Dementia: Review and Meta-Analysis. J Alzheimers Dis, 2017. 56(1): p. 215-228.

15. Huang, C.Q., et al., Association of cognitive impairment with serum lipid/lipoprotein among Chinese nonagenarians and centenarians. Dement Geriatr Cogn Disord, 2009. 27(2): p. 111-6.

16. Reitz, C., et al., Plasma lipid levels in the elderly are not associated with the risk of mild cognitive impairment. Dement Geriatr Cogn Disord, 2008. 25(3): p. 232-7.

17. He, Y., et al., Cohort Profile: The China Hainan Centenarian Cohort Study (CHCCS). Int J Epidemiol, 2018.

18. Yao, Y., et al., The prevalence of depressive symptoms in Chinese longevous persons and its correlation with vitamin D status. BMC Geriatr, 2018. 18(1): p. 198.

19. Tombaugh, T.N. and N.J. McIntyre, The mini-mental state examination: a comprehensive review. J Am Geriatr Soc, 1992. 40(9): p. 922-35.

20. Vermeulen, J., et al., Predicting ADL disability in community-dwelling elderly people using physical frailty indicators: a systematic review. BMC Geriatr, 2011. 11: p. 33.

21. Bates, K.A., et al., Serum high-density lipoprotein is associated with better cognitive function in a cross-sectional study of aging women. Int J Neurosci, 2017. 127(3): p. 243-252.

22. Sacco, R.L., et al., High-density lipoprotein cholesterol and ischemic stroke in the elderly: the Northern Manhattan Stroke Study. Jama, 2001. 285(21): p. 2729-35.

23. Formiga, F., et al., Serum high-density lipoprotein cholesterol levels correlate well with functional but not with cognitive status in 85-year-old subjects. J Nutr Health Aging, 2012. 16(5): p. 449-53.

24. Landi, F., et al., Serum high-density lipoprotein cholesterol levels and mortality in frail, communityliving elderly. Gerontology, 2008. 54(2): p. 71-8.

25. Sun, Y., et al., Serum high-density lipoprotein cholesterol is a protective predictor of executive function in older patients with diabetes mellitus. J Diabetes Investig, 2018.

26. Badimon, L. and G. Vilahur, LDL-cholesterol versus HDL-cholesterol in the atherosclerotic plaque: inflammatory resolution versus thrombotic chaos. Ann N Y Acad Sci, 2012. 1254: p. 18-32.

27. Rubins, H.B., et al., Gemfibrozil for the secondary prevention of coronary heart disease in men with low levels of high-density lipoprotein cholesterol. Veterans Affairs High-Density Lipoprotein Cholesterol Intervention Trial Study Group. N Engl J Med, 1999. 341(6): p. 410-8.

28. Olesen, O.F. and L. Dag $\varnothing$, High density lipoprotein inhibits assembly of amyloid beta-peptides into fibrils. Biochem Biophys Res Commun, 2000. 270(1): p. 62-6.

29. Kravitz, E., J. Schmeidler, and M.S. Beeri, Cognitive decline and dementia in the oldest-old. Rambam Maimonides Med J, 2012. 3(4): p. e0026. 
30. Corrada, M.M., et al., Dementia incidence continues to increase with age in the oldest old: the 90+ study. Ann Neurol, 2010. 67(1): p. 114-21.

31. Slavin, M.J., H. Brodaty, and P.S. Sachdev, Challenges of diagnosing dementia in the oldest old population. J Gerontol A Biol Sci Med Sci, 2013. 68(9): p. 1103-11.

32. Lucca, U., et al., Prevalence of dementia in the oldest old: the Monzino 80-plus population based study. Alzheimers Dement, 2015. 11(3): p. 258-70 e3. 